\title{
Interaction between dielectric particles enhances the Q-factor
}

\author{
Evgeny Bulgakov $^{1,2}$, Konstantin Pichugin ${ }^{1}$, Almas Sadreev ${ }^{1}$ \\ ${ }^{1}$ Kirensky Institute of Physics, Federal Research Center KSC Siberian Branch, RAN, Krasnoyarsk 660036, Russia \\ ${ }^{2}$ Reshetnev Siberian State University of Science and Technology, 660037, Krasnoyarsk, Russia \\ corresponding author, E-mail: almasetnp.krasn.ru
}

\begin{abstract}
We study behavior of resonant modes with a distance between two dielectric resonators shaped as cylinders and disks. We reveal two basic scenarios of evolution of resonances with the distance between the cylinders. For larger distances and respectively weaker interaction of particles the resonances are bound around the resonances of isolated resonators and evolve by spiral way. For shorter distances and respectively stronger interaction the resonances bypass the isolated resonances. Both scenarios demonstrate considerable enhancement of the $Q$ factor compared to the case of isolated particle.
\end{abstract}

\section{Introduction}

It is rather challenging for optical resonators to support resonances of simultaneous subwavelength mode volumes and high $Q$ factors. The traditional way for increasing the $Q$ factor of optical cavities is a suppression of leakage of resonance mode into the radiation continua. That is achieved usually by decreasing the coupling of the resonant mode with the continua by the use of metals, photonic band gap structures, or whispering-gallery-mode resonators. All of these approaches lead to reduced device efficiencies because of complex designs, inevitable metallic losses, or large cavity sizes. On the contrary, all-dielectric subwavelength nanoparticles have recently been suggested as an important pathway to enhance capabilities of traditional nanoscale resonators by exploiting the multipolar Mie resonances being limited only by radiation losses $[1,2]$.

The decisive breakthrough came with the paper by Friedrich and Wintgen [3] which put forward the idea of destructive interference of two neighboring resonant modes leaking into the continuum. Based on a simple generic twolevel model they formulated the condition for the bound state in the continuum (BIC) as the state with zero resonant width for crossing of eigenlevels of the cavity or avoided crossing of resonances. This principle was later explored in open plane wave resonator where the BIC occurs in the vicinity of degeneracy of the closed integrable resonator [4].

However, these BICs exist provided that they embedded into a single continuum of propagating modes of a directional waveguide. In photonics the optical BICs embedded into the radiation continuum can be realized by two ways. The first way is realized in an optical cavity coupled with the continuum of $2 \mathrm{~d}$ photonic crystal ( $\mathrm{PhC})$ waveguide [5] that is an optical variant of microwave system [4]. Alternative way is the use periodic $\mathrm{PhC}$ systems (gratings) or arrays of dielectric particles in which resonant modes leak into a restricted number of diffraction continua $[6,7,8,9,10]$. Although the exact BICs can occur only in infinite periodical arrays, the finite arrays demonstrate resonant modes with the very high $Q$ factor which grows quadratically [11] or even cubically [12] with the number of particles (quasiBICs). Even arrays of five dielectric particles demonstrate the $Q$ factor exceeding the $Q$ factor of individual particle by six orders in magnitude [13].

Isolated subwavelength high-index dielectric resonators are more advantageous from an applied point of view to achieve high $Q$ resonant modes (super cavity modes) $[2,14,15]$. Such super cavity modes originate from avoided crossing of the resonant modes, specifically the Mie-type resonant mode and the Fabry-Pérot resonant mode under variation of the aspect ratio of the dielectric disk which could result in a significant enhancement of the $Q$ factor. It is worthy also to notice the idea of formation of long-lived, scar like modes near avoided resonance crossings in optical deformed microcavities [16]. The dramatic $Q$ factor enhancement was predicted by Boriskina $[17,18]$ for avoided crossing of very highly excited whispering gallery modes in symmetrical photonic molecules of dielectric cylinders on a surface. In the present paper we consider a similar way to enhance the $Q$ factor by variation of the distance between two identical dielectric cylinders and coaxial disks as sketched in Fig. 1. As different from papers $[16,17,18,19,20]$ we consider the avoided crossing of low excited resonant modes (monopole, dipole and quadruple) with variation of the distance between two cylinders. Because of lifting of the axial symmetry many Mie resonances contribute into the resonances of two cylinders which show two basic scenarios of evolution with the distance, bound to the Mie resonances and unbound. The same scenarios show the resonances of two coaxial dielectric disks however with those difference that for small distance between them there might be avoided crossing of different resonances of the isolated disk. 

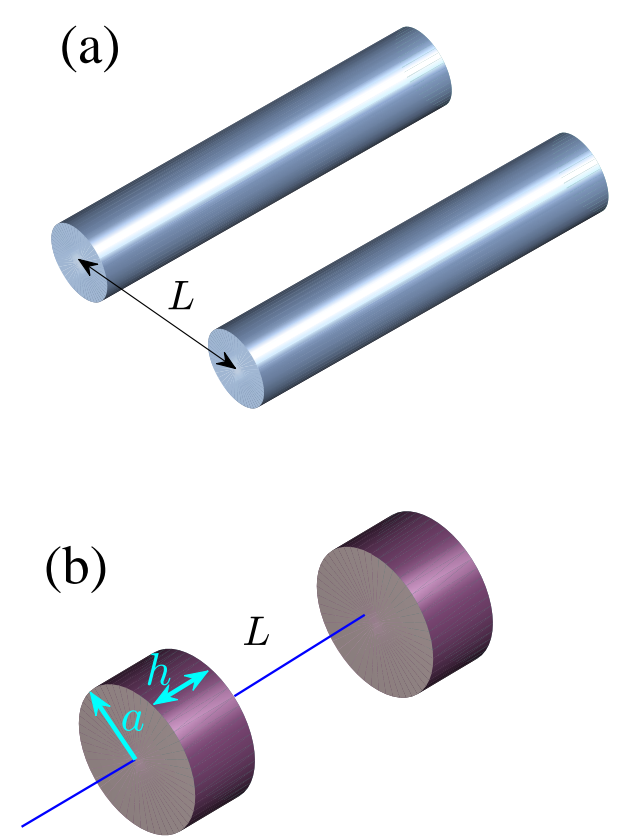

Figure 1: Two parallel dielectric cylinders (a) and coaxial disks (b)

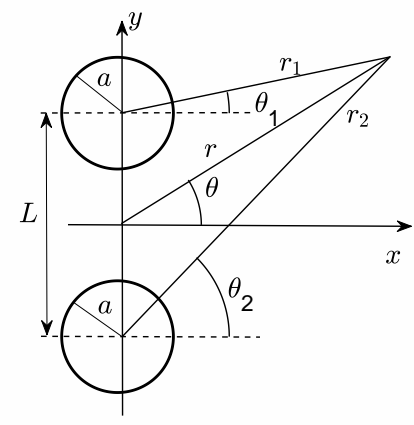

Figure 2:

\section{Avoided crossings under variation of the distance between two cylinders}

The problem of scattering of electromagnetic waves from two parallel infinitely long dielectric cylinders sketched in Figs. 2 was solved long time ago in papers [21, 22, 23]. The solutions for electromagnetic field, the component of electric field directed along the cylinders $\psi=E_{z}$ outside the cylinders are given by

$$
\begin{aligned}
& \psi_{1}=\sum_{n} A_{1 n} H_{n}^{(1)}\left(k r_{1}\right) e^{i n \theta_{1}}, \\
& \psi_{2}=\sum_{n} A_{2 n} H_{n}^{(1)}\left(k r_{2}\right) e^{i n \theta_{2}} .
\end{aligned}
$$

Inside the cylinders we have

$$
\begin{gathered}
\psi_{1}=\sum_{n} B_{1 n} J_{n}\left(\sqrt{\epsilon} k r_{1}\right) e^{i n \theta_{1}}, \\
\psi_{2}=\sum_{n} \sum_{n} B_{2 n} J_{n}\left(\sqrt{\epsilon} k r_{2}\right) e^{i n \theta_{2}} .
\end{gathered}
$$

By means of the Graf formula [24]

$$
\begin{aligned}
& H_{n}^{(1)}\left(k r_{1}\right) e^{i n \theta_{1}}=\sum_{m} i^{n-m} H_{m-n}^{(1)}(k L) J_{m}\left(k r_{2}\right) e^{i m \theta_{2}},(5) \\
& H_{n}^{(1)}\left(k r_{2}\right) e^{i n \theta_{2}}=\sum_{m} i^{n-m} H_{m-n}^{(1)}(k L) J_{m}\left(k r_{1}\right) e^{i m \theta_{1}},(6)
\end{aligned}
$$

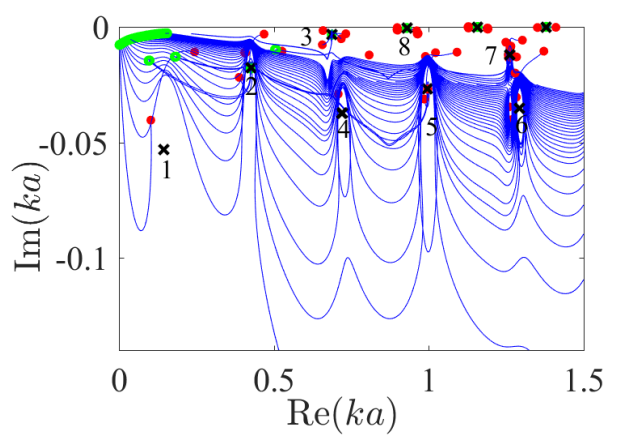

Figure 3: The behavior of resonant frequencies vs the distance between cylinders. Open green circles correspond $L=1000 a$, closed red circles correspond to minimal distance $L=2 a$, where $a$ is the radius of cylinders and black crosses are the Mie resonances shown in the next Fig. 4.

the total field $\psi=\psi_{i n c}+\psi_{1}+\psi_{2}$ can be written completely in the coordinate system of either cylinder.

Applying the boundary conditions at $r_{j}=a$ leads to $[21,23]$

$$
\begin{aligned}
& A_{1 n}=i^{n} S_{n}(k) \sum_{m} i^{-m} H_{n+m}(k L) A_{2 n}, \\
& A_{2 n}=i^{n} S_{n}(k) \sum_{m} i^{-m} H_{n+m}(k L) A_{1 n},
\end{aligned}
$$

where $S_{n}$ are the scattering matrix amplitudes for the isolated cylinder

$$
S_{n}(k)=\frac{\sqrt{\epsilon} J_{m}^{\prime}(\sqrt{\epsilon} k a) J_{m}(k)-J_{m}^{\prime}(k) J_{m}(\sqrt{\epsilon} k)}{H_{m}^{(1)^{\prime}}(k) J_{m}(\sqrt{\epsilon} k)-\sqrt{\epsilon} J_{m}^{\prime}(\sqrt{\epsilon} k) H_{m}^{(1)}(k)} .
$$

The resonances are given by the complex roots of the following equation

$$
\operatorname{Det}\left[\hat{M}^{2}-I\right]=0
$$

where matrix elements $\hat{M}$ is given by Eq. (7) and equal

$$
M_{m n}=S_{m}(k) i^{m-n} H_{m+n}(k L)
$$

and $I$ is the unit matrix. In what follows the dimensionless complex eigenvalues $k$ are measured in terms of $c / a$ where $c$ is the light velocity and $a$ is the radius of cylinders and $L$ is measured in terms of $a$. .

In general Eq. (9) has an infinite number of complex resonant frequencies $k_{n}=k_{n r}-i \gamma_{n}$. The total view of the dependence of resonant eigenvalues is shown in Fig. 3 which demonstrates complicated evolution of resonant poles against the distance between the cylinders. First of all one can see the major part of the resonances are unbound bypassing the Mie resonances of the isolated cylinder marked by crosses which are collected in Fig. 4. Second, there are a small part of resonances which are bound around the Mie resonances with smaller imaginary parts. 


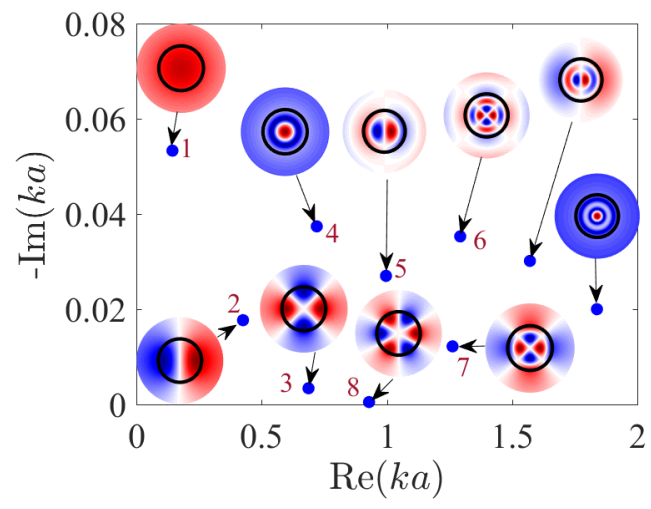

Figure 4: The Mie resonant complex eigenfrequencies (close circles) and corresponding resonant modes (the component $E_{z}$ ) of isolated cylinder.

Let us consider the asymptotic behavior of resonances for $L \rightarrow \infty$. By use of asymptotical behavior of the Hankel functions [25] we have for matrix (10) the following

$$
M_{m n} \sim \sqrt{\frac{2}{\pi k L}} e^{i(k L-\pi / 4)} S_{m}(k)(-1)^{n} .
$$

Let us take the eigenvector of matrix $\widehat{M} \vec{\psi}^{+}=$ $\left(\psi_{1}, \psi_{2}, \psi_{3}, \ldots\right)$. Then Eq. (9) takes the following form

$$
\sqrt{\frac{2}{\pi k L}} e^{i k L} S_{m}(k) \sum_{n}(-1)^{n} \psi_{n}= \pm \psi_{m},
$$

which has the solution provided that

$$
\sqrt{\frac{2}{\pi k L}} e^{i k L} \sum_{n}(-1)^{n} S_{n}(k)= \pm 1 .
$$

For the absolute value we have

$$
\frac{2 e^{2 \gamma_{n} L}}{\pi\left|k_{n}\right| L}=\frac{1}{\left|\sum_{n}(-1)^{n} S_{n}(k)\right|^{2}}
$$

where $\gamma_{n}=-\operatorname{Im}\left(k_{n}\right)$. The resonances of the isolated cylinder are given by poles of the $\mathrm{S}$-matrix, i.e., by equation $\frac{1}{S_{m}(k)}=0$. Therefore from (14) it follows that, first, the resonances of two cylinders do not converge to the resonances of the isolated cylinder. Second, because of finite value of right hand side of Eq. (14) the line widths and resonance positions limit to zero at $L \rightarrow \infty$. Therefore we can conclude that the resonances of two dielectric cylinders do NOT limit to the Mie resonances of the isolated cylinders at $L \rightarrow \infty$. The reason is related to to the exponential factor $\exp \left(\gamma_{n} L\right)$ of the resonant modes (the Gamov states) at large distances between the cylinder.

Next, we consider the behavior of some typical resonances in Fig. 4 in detail from the limiting case $L=2 a$ to $L=1000 a$. Due to the symmetry of the system relative to $x \rightarrow-x$ and $y \rightarrow-y$ the resonant modes can be classified as $\psi_{\sigma ; \sigma^{\prime}}$ where the indices $\sigma=s, a$ correspond to
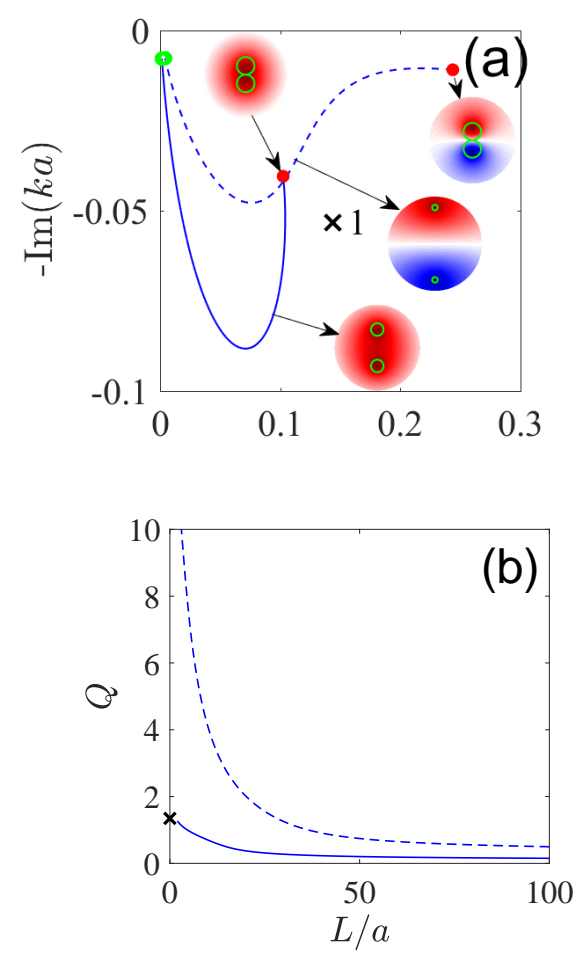

Figure 5: Evolution of resonant frequencies and monopole resonant modes $\psi_{s, s / a}$ (a) and the $Q$ factors (b) with the distance between the cylinders. Solid/dash lines show symmetric/antisymmetric resonant frequencies. Closed circles correspond to $L=2 a$, open circles correspond to $L=$ $1000 a$ and cross corresponds to the monopole Mie resonance 1 .

the symmetric and antisymmetric modes respectively. We start with the first two lowest symmetric and antisymmetric monopole resonant modes $\psi_{s ; s / a}$.

The quality factor is most commonly defined as the ratio of the stored energy in the resonator over the dissipated energy per optical cycle $Q=k W / P_{\text {loss }}$ where $W$ is the stored energy, and $P_{\text {loss }}$ represents the power losses. When the stored energy dissipates through radiation of the resonant mode with complex eigenfrequency $k_{n}=k_{n r}-i \gamma_{n}$ we observe exponential decay of the stored energy $W=W_{0} \exp \left(-2 \gamma_{n} t\right)$. Therefore the radiation power $P_{\text {loss }}=-d W / d t=2 \gamma_{n} W$ that allows to find the quality factor directly from the eigenfrequency as $Q=k_{n r} / 2 \gamma_{n}$. Fig. 5 (b) shows that the $Q$ factor of the antisymmetric monopole resonance exceeds the $Q$ factor of the isolated cylinder by one order in magnitude. The reason for that follows from the Mie resonances shown in Fig. 4. As seen from Fig. 5 (a) at the closest distance between the cylinders $L=2 a$ the symmetric resonant mode becomes the monopole mode with corresponding $Q$ factor close to the $Q$ factor of isolated cylinder which is rather low as marked by cross in Fig. 5 (b). At the same time the antisymmetric mode of two cylinders at $L=2 a$ becomes 

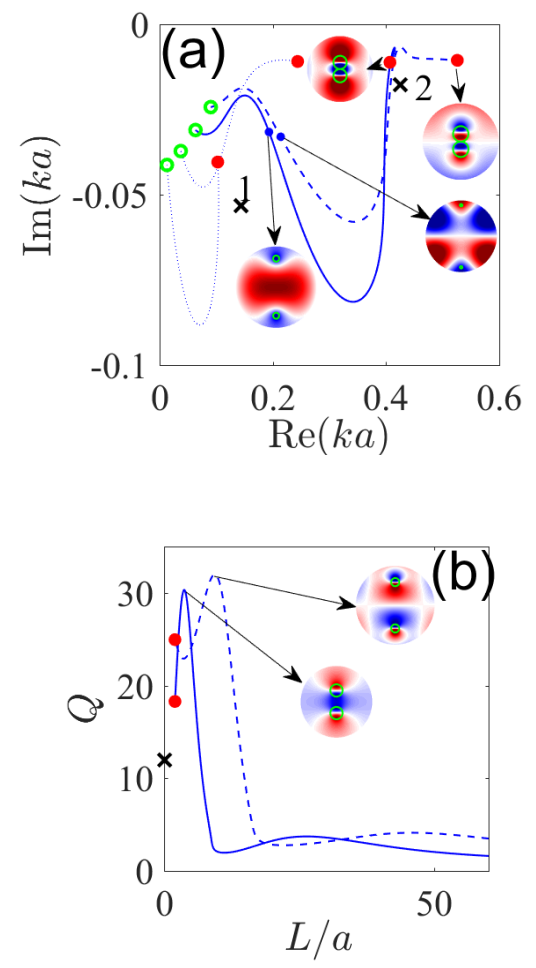

Figure 6: The same as in Fig. 5 for the dipole resonant modes $\psi_{s ; s / a}$ symmetric relative to $x \rightarrow-x$. Cross marks the Mie resonance (a) and respectively $Q$ factor of the isolated cylinder (b).

the dipole resonance which as seen from Fig. 4 has the $Q$ factor exceeding the $Q$ factor of the monopole Mie resonance by one order in magnitude. The next resonances illustrate that the evolution of resonances strongly depend on interaction between the cylinders via the radiating Mie resonances. The general expressions and physical origin of the coupling of dielectric resonators was considered in Refs. [26, 27, 28]. The coupling constant can be written as [27]

$$
\kappa=\int d v[\epsilon(\vec{r})-1] \vec{E}_{1}^{*} \vec{E}_{2}
$$

where $\vec{E}_{1,2}$ are normalized solutions by the factor $\sqrt{\int \epsilon(\vec{r})\left|\vec{E}_{1,2}\right|^{2} d v}$. Here the indices 1 and 2 imply the resonant modes of the corresponding dielectric particles. The coupling constant is determined by overlapping of resonant modes which in turn depend on the distance between the particles and prevailing direction of radiation of the modes.

That conclusion is well illustrated by the Mie dipole resonant modes which are degenerate. The first dipole Mie resonant mode, symmetric relative to $x \rightarrow-x$, radiates prevalently towards the neighboring cylinder as shown in insets in Fig. 6 while the second antisymmetric dipole Mie resonant mode radiates away from the neighboring cylinder as shown in insets of Fig. 7. As a result the interaction in (a)

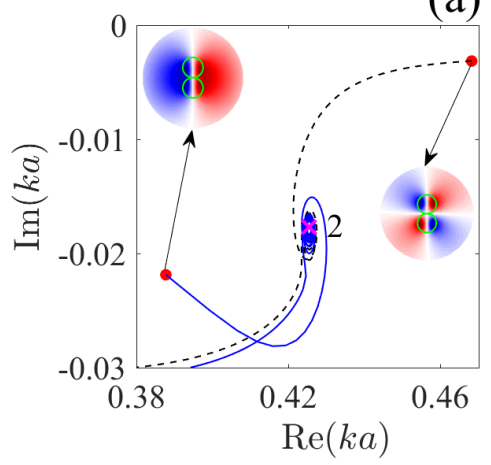

(b)

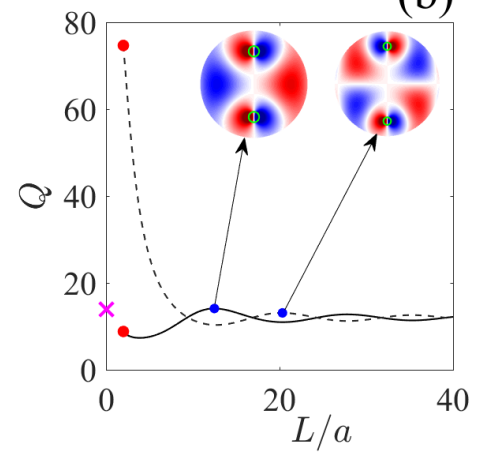

Figure 7: The same as in Fig. 6 for the dipole resonant modes $\psi_{a ; s / a}$ antisymmetric relative to $x \rightarrow-x$.

former case turns out stronger compared to the latter case as it follows from Eq. (15). That explains why the evolution of resonances shown in Fig. 6 (a) is similar to the case of interaction via the monopole resonant modes in Fig. 5 while the evolution of resonances in Fig. 6 is bound to the Mie dipole resonance 2 as seen from Fig. 7 (a). Respectively the gain in the $Q$ factor in the former case is smaller than in the latter case as seen from Fig. 7 (b).

With further increase of the distance $L$ the resonance bypasses the monopole Mie resonance 1 of the isolated cylinder. As a result the solution of the Maxwell equations becomes close to the resonant mode $\psi_{s ; s / a}$. That rule for the solutions when the resonances bypass the Mie resonances is fulfilled for all other resonances as one can see from Fig. 8. Similar evolution scenarios can be observed for the quadruple resonances shown in Fig. 9 (a). In this case the Mie resonant mode radiates from one cylinder to another that gives rise to interaction between cylinders stronger than the case of the modes $\psi_{a ; s / a}$ shown in Fig. 9 (c). Both cases result in interaction weaker than the case of dipole Mie resonances. As a result in both cases one can observe that the resonances are bound around the Mie quadruple resonance 3 shown in Fig. 4 for distances from $L=2 a$ to $L=350 a$. However, in the first case of stronger interaction between the cylinders the resonances $\psi_{s ; s / a}$ go away from the initial Mie resonance 3 and consequently go to zero bypassing the Mie resonance 2 as seen in 
(a)

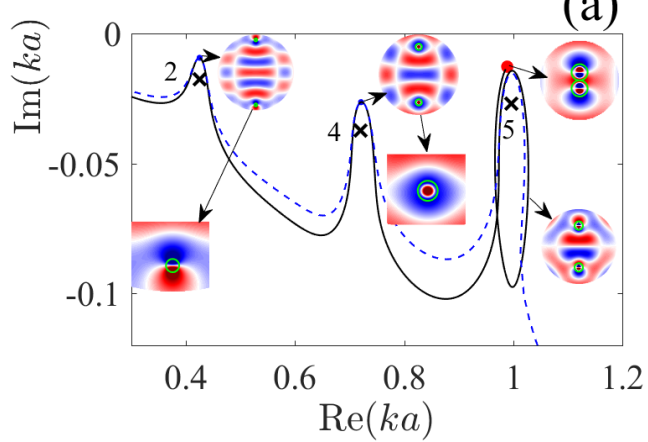

(b)

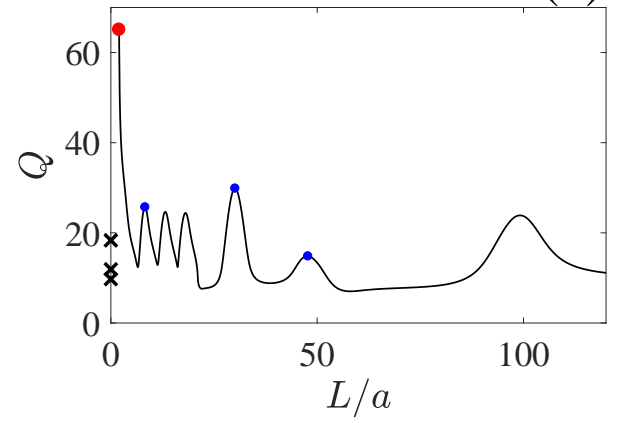

Figure 8: Evolution of symmetric and antisymmetric hybridizations of higher Mie dipole resonant modes (a) and respective $Q$ factors (b) with the distance.

Fig. 4. meanwhile in the second case of weaker interaction the resonances $\psi_{a ; s / a}$ remain bound around the quadruple Mie resonance 3 as shown in Fig. 9 (c). The dependence of the $Q$ factor on $L$ is shown in Fig. 9 (b) and (d).

\section{Avoided crossings in system of two coaxial disks}

The system of two parallel dielectric cylinders has advantage of analytical consideration of the resonances by means of Eqs. (9) and (10). However in actual design the cylinders will have finite length that substantially complicates equations for the resonant frequencies [29]. The system of two coaxial disks sketched in Fig. 1 (b) is practically more preferable. On the one hand the system preserves the axial symmetry that allows to separate the azimuthal angle $\phi$ and consider the resonances with fixed azimuthal index $m$. Experimentally disks are to be fixed by a special holder. However in the $\mathrm{GHz}$ range the holder from a Styrofoam material with a permittivity close 1.1 compared to the permittivity of ceramic disks around 40 only slightly disturb the axial symmetry [11]. If to restrict ourselves by by the sector $m=0$ the resonances can be excited by coaxial loop antenna [11] that is the present subject of consideration. For the case $m=0$ the solutions are separated by polarization with $H_{z}=0$ (E modes) and $E_{z}=0$ (H modes). In what follows we consider the H-modes. In general the resonant modes and their eigenfrequencies are given by solving the (a)

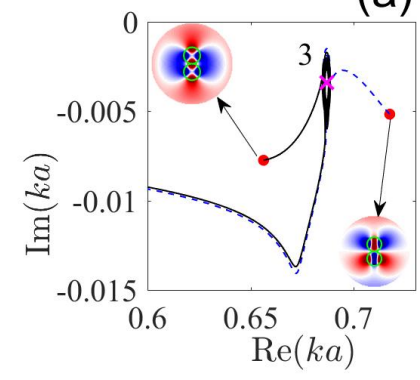

(b)
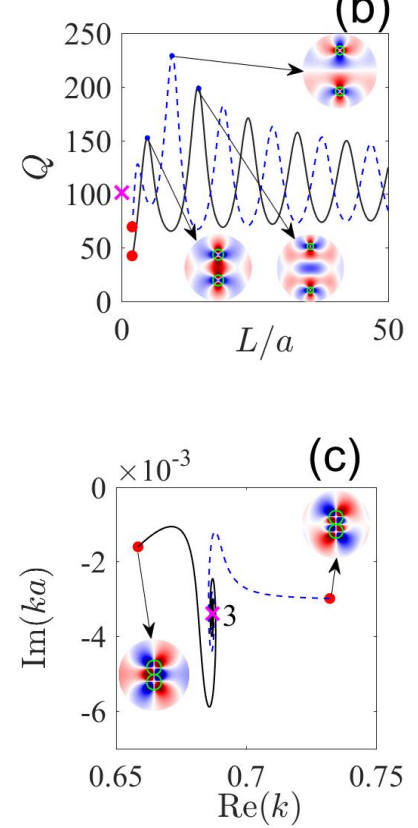

(d)

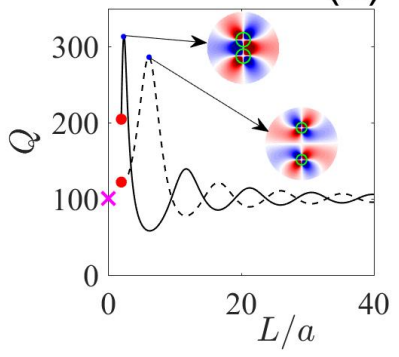

Figure 9: The evolution of quadruple resonant modes under vs the distance. Crosses mark the Mie resonances shown in Fig. 4. Red closed circle marks the case of the smallest distance and open green circle marks the distance $L=1000 \mathrm{a}$.

time-harmonic source-free Maxwell's equations [30, 31]

$$
\left(\begin{array}{cc}
0 & \frac{i}{\epsilon} \nabla \times \\
-i \nabla \times & 0
\end{array}\right)\left(\begin{array}{c}
\mathbf{E}_{n} \\
\mathbf{H}_{n}
\end{array}\right)=k_{n}\left(\begin{array}{c}
\mathbf{E}_{n} \\
\mathbf{H}_{n}
\end{array}\right)
$$

where $\mathbf{E}_{n}$ and $\mathbf{H}_{n}$ are the EM field components defined in Ref. [31] as quasinormal modes which are also known as 
(a)

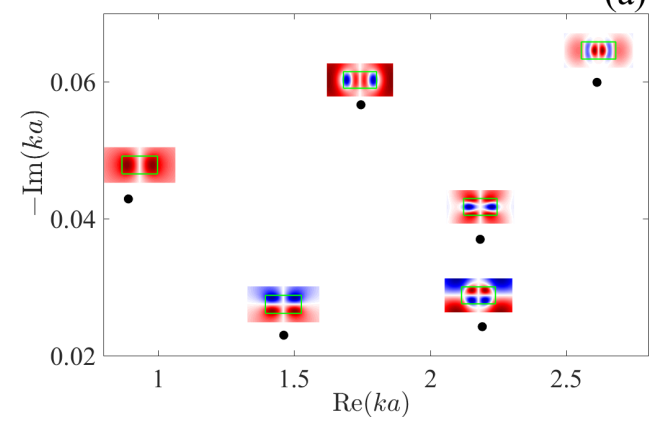

Figure 10: The resonant eigenfrequencies (close circles) and corresponding resonant modes (the component $E_{\phi}$ of isolated dielectric disk with the height $h=a$ and permittivity $\epsilon=40$.
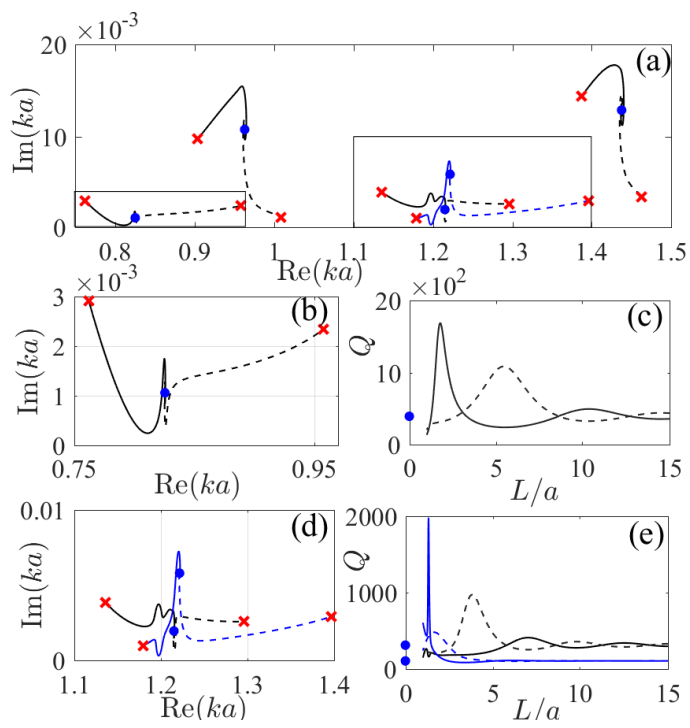

Figure 11: (a) The behavior of resonant eigenfrequencies under variation of the distance between the disks $L$ with the $\epsilon=40, a=h$. (b) and (d) zoomed areas highlighted in (a) with symmetric (solid lines) and antisymmetric (dash lines) resonant modes. (c) and (e) show the behavior of the $Q$ factor vs the distance for corresponding insets at the left. Closed circles mark the eigenfrequencies of isolated disks and respectively the $Q$ factors while crosses mark the limiting case $L=h$ when two disks stick together.

resonant states [32, 33] or leaky modes [34]. It is important that they can be normalized and the orthogonality relation can be fulfilled by the use of perfectly matched layers (PMLs) [31]. With the exception of very restricted number of symmetrical particles Eq. (16) can be solved only numerically. The eigenfrequencies are complex $k_{n} a=\omega_{n}+i \gamma_{n}$ where $a$ is the disk radius. In what follows the light velocity is taken unit. Fig. 10 shows resonant frequencies of the isolated disk complimented by insets with the resonant modes (only the component $E_{\phi}$ is shown). There are modes with nodal surfaces crossing the $\mathrm{z}$-axis and the modes with nodal surfaces crossing the plane $z=0$. They correspond to the
Fabry-Perot resonant modes and the radial Mie modes by the terminology introduced in paper [14].

Fig. 11 shows the solutions of Eq. (16) for the case of two coaxial dielectric disks as dependent on the distance $L$ between the disks. The necessity to use PMLs restricts the distance between the disks which is to be considerably less than the distance between the PMLs in the z-direction.

In spite of an illusive complexity in Fig. 11 the zoomed pictures reveal remarkably simple behavior of resonant frequencies in the form of a spiral convergence of the eigenfrequencies to the resonant frequencies of the isolated disk marked by closed circles. However, when the disks approach close enough to each other the spiralling behavior is replaced by strong repulsion of resonant frequencies because of interaction enhancement.

In order to quantitatively evaluate this interaction we start consideration with an isolated disk for which the matrix of derivatives in Eq. (16) becomes diagonal with the complex eigenfrequencies $k_{n}$ in the eigenbasis presented in Fig. 10. It is reasonable to assume that for enough separation between disks the matrix is still diagonal with pairs of degenerate $k_{n}$ shown in Fig. 11 by blue closed circles. As the distance between the disks is reduced the interaction between the disks via the resonant modes splits the degenerate resonant modes $k_{n}$ giving rise to an avoided crossing. We also assume also that the value of splitting much less than the distance between the different $k_{n}$. These assumptions are justified numerically as shown in insets of Fig. 11, however, for only in definite domains of the frequency $k$ around the resonances of the isolated disk where spiral behavior of the resonant frequencies takes place. In the framework of these assumptions we can use two-level approximation for the Hamiltonian matrix in Eq. (16) for each resonance $k_{n}$ $[16,15,31]$

$$
H_{e f f}^{(n)}=H_{e f f}^{(0)}+V=\left(\begin{array}{cc}
k_{n} a & 0 \\
0 & k_{n} a
\end{array}\right)+\left(\begin{array}{cc}
u_{n} & v_{n} \\
v_{n} & u_{n}
\end{array}\right),
$$

where $v_{n}$ is responsible for interaction between the disks via the resonant modes while $u_{n}$ is the result of the backscattering by the first disk. Therefore one can expect that $\arg \left(v_{n}\right)=\omega_{n} L / a, \arg \left(u_{n}\right)=2 \omega_{n} L / a$. Fig. 12 shows the behavior of the absolute value and phase both of the matrix elements. The matrix elements $v_{n}$ and $u_{n}$ can be easily found from numerically calculated resonances shown in Fig. 11

$$
k_{a, s}^{(n)} a=k_{n} a+u_{n} \pm v_{n},
$$

as $v_{n}=\frac{k_{s}^{(n)}-k_{a}^{(n)}}{2}, u_{n}=\frac{k_{s}^{(n)}+k_{a}^{(n)}}{2}-k_{n}$. From Fig. 12 one can evaluate that the interaction term in (17)

$$
v_{n} \sim \frac{e^{i k_{n} L}}{L^{2}}, u_{n} \sim \frac{e^{2 i k_{n} L}}{L^{4}} .
$$

The distance behavior (19) is observed with a good accuracy for all resonances shown in Fig. 12, however, for only spiral convergence of the resonances. Numerically calculated behavior of the matrix elements $v_{n}$ and $u_{n}$ for $n=2$ is shown in Fig. 12. In spiralling around the resonances 

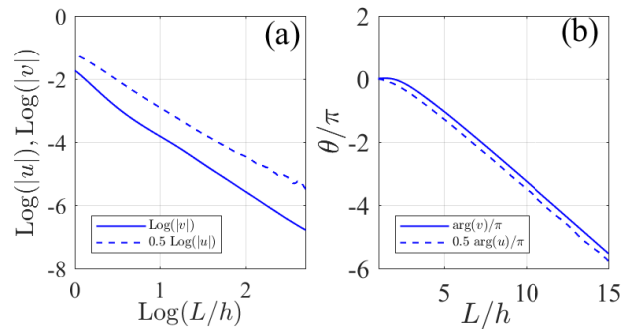

Figure 12: Dependence of the matrix elements $v_{n}$ and $u_{n}$ on the distance between disks $L$.

of the isolated disk the hybridized resonant eigenmode is given by symmetric and antisymmetric combinations of the resonant modes of the isolated disk

$$
\psi_{s, a}(\vec{r})=\psi_{n}\left(\vec{r}-\frac{1}{2} L \vec{e}_{z}\right) \pm \psi_{n}\left(\vec{r}+\frac{1}{2} L \vec{e}_{z}\right)
$$

where $\vec{e}_{z}$ is the unit vector along the z-axis and $\psi_{n}(\vec{r})$ are the corresponding resonant modes of the isolated disk shown in the insets in Fig. 10.

At first the resonant frequencies slowly spiral away from the limiting point given by $k_{n}$. Respectively the $Q$ factor in Fig. 11 (c) demonstrates oscillating behavior exceeding the $Q$ factor of the isolated disk a few times. As the disks approach each other the spiral behavior of the pair of resonances $k_{s, a}^{(n)}$ is replaced by strong repulsion as shown Fig. 11 (b). Fig. 11 (d) shows a remarkable feature caused by the avoided crossing of resonances with different $n$. To be specific there is an avoided crossing of symmetric resonances $k_{s}^{(2)}$ and $k_{s}^{(5)}$ according to enumeration in Fig. 10. Because of the same symmetry these resonances undergo typical avoided crossing with a considerable decrease of the imaginary part of the resonant frequency and correspondingly enhancement of the $Q$ factor by one order in magnitude. Respectively the two-mode approximation (17) breaks down.

It is interesting to trace the behavior of resonances and the $Q$ factors for the aspect ratio $h=1.0395 a$ and $\epsilon=40$ for which the isolated disk shows the maximal $Q$ factor. The results are presented in Fig.13. One can see that with decrease of the distance between the disks we have the same spiralling behavior of the hybridized resonances around the resonances of the isolated disks which is terminated by strong repulsion of the symmetric and antisymmetric resonances for $L \rightarrow h$. However, we don't see a pronounced effect of the avoiding crossing of hybridized resonances with different $n$ and respectively have no enhancement of the $Q$ factor by one order in magnitude as it was achieved for the aspect ratio $a / h=1$ (see Fig. 12 (e)). With account of material losses the $Q$ factor is to be corrected via the relation $\frac{1}{Q}=\frac{\operatorname{Re}\left(k_{n} a\right)}{2 \operatorname{Im}\left(k_{n} a\right)}+\tan \delta$ where $\tan \delta=\epsilon^{\prime \prime} / \epsilon^{\prime}$ is result of material losses given by the imaginary part of the permittivity $\epsilon=\epsilon^{\prime}-i \epsilon^{\prime \prime}$. For ceramic disks with $\epsilon=40, \tan \delta=2.5 \times 10^{-4}$ and therefore $\epsilon^{\prime \prime}=0.01$ [11]. In Fig. 13 (c) and (e) we show that even small material losses become important for enhancement of the $Q$ factor.
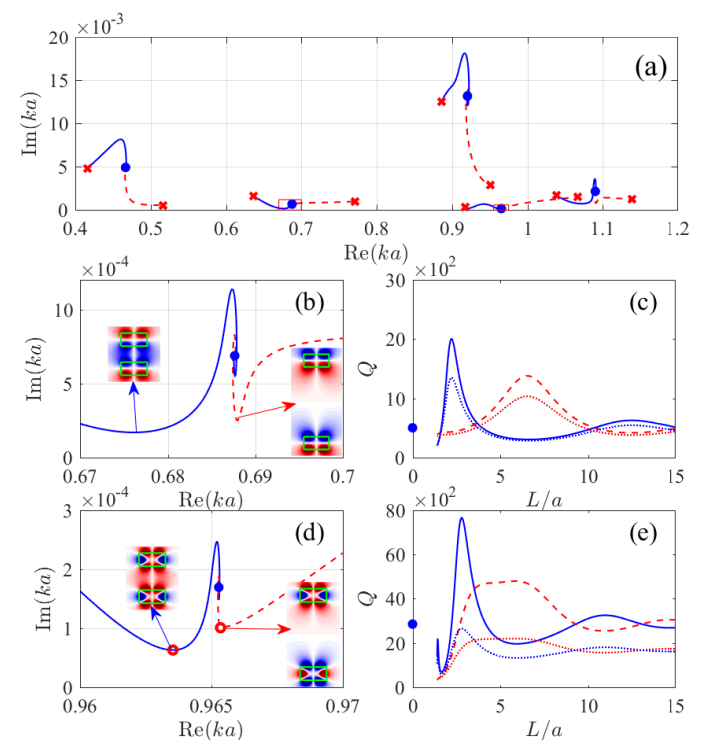

Figure 13: (a) Behavior of resonant eigenfrequencies under variation of the distance between the disks $L$ with $\epsilon=40$ (ceramics in THz range) and the aspect ratio $a / h=0.7$. (b) and (d) zoomed areas highlighted in (a) by red rectangles with symmetric (solid lines) and antisymmetric (dash lines) hybridized resonant modes (20) of. (c) and (e) show behavior of the $\mathrm{Q}$ factor vs the distance. Closed circles mark the eigenfrequencies of isolated disks and respectively the $Q$ factors while crosses mark the limiting case $L=a$ when the two disks stick together. Dotted lines show the behavior of the $Q$ factors with account of material losses in ceramic disks with $\tan \delta=2.5 \times 10^{-4}$ in THz range [11]

Up to now we considered the permittivity $\epsilon=40$ and $a=1 \mathrm{~cm}$ (ceramic disks) that enters the resonant frequencies into the $\mathrm{THz}$ range. Finally, we consider $\epsilon=12$ (silicon disks) and $a=h=1 \mu \mathrm{m}$ with the resonant frequencies in the infrared range $\lambda=1.2 \mu \mathrm{m}$ where material losses are extremely small [35]. Results of computations are presented in Fig. 14 which shows that there is no qualitative difference between the ceramic disks with $\epsilon=40$ and silicon disks with $\epsilon=12$. Similar to Fig. 11 and Fig. 13 we observe spiral behavior of the resonant frequencies for the enough distance between the disks. But what is more remarkable we also observe an avoided crossing of the resonances with different indices as shown in Fig. 14 (d) with corresponding strong enhancement of the $Q$ factor by one order in magnitude (Fig. 14 (e)).

\section{Conclusions}

For the isolated dielectric cylinder we have well known Mie resonances specified by azimuthal index $m=$ $0, \pm 1, \pm 2, \ldots$ (monopole, dipole, quadruple etc resonances) due to axial symmetry. Two parallel cylinders have no axial symmetry and therefore the solutions of the homogeneous Maxwell equations are given by series of the Bessel (inside) or Hankel (outside cylinders) functions in 


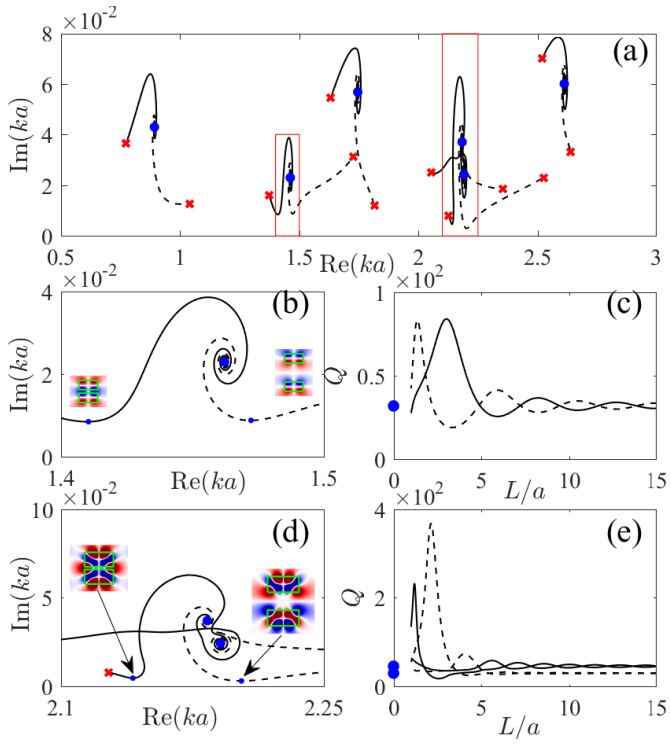

Figure 14: (a) Behavior of resonant eigenfrequencies under variation of the distance between the disks $L$ with $\epsilon=12$ (silicon in optical range) and aspect ratio $a / h=1$. (b) zoomed area highlighted in (a) with symmetric (solid lines) and antisymmetric (dash lines) hybridization (20) of resonant modes of the isolated disk. (c) shows behavior of the $Q$ factor vs the distance for corresponding insets at the left. Closed circles mark the eigenfrequencies of isolated disks and respectively the $Q$ factors while crosses mark the limiting case $L=a$ when two disks stick together.

$m$. By the use of Graf formula the coefficients in series satisfy linear algebraic equations and can be easily found $[21,22,23]$. However, there were no studies on the behavior of resonances of two cylinders in dependence on the distance between them except the studies of the $Q$ factor for extremely highly excited resonances, whispering gallery modes by Boriskina $[17,18]$. The study presented in this paper reveals surprisingly rich evolution of the resonances with the distance that can be described by two scenarios. In the first scenario the resonances subsequently bypass the Mie resonances. Each time when the resonance is close to a Mie resonance the resonant mode inside the cylinders takes the field profile of the corresponding Mie resonant mode. At the same time the $Q$ factor achieves maximal magnitudes. It is worthy to notify recent publication by Abdrabou and Ya Yan Lu [36] in which exceptional points for resonant states were achieved for variation of the distance between dielectric cylinders.

The evolution of resonances bound by the Mie resonances follows the second scenario and typical for the higher resonances with $n=2,3, \ldots$ (where the index enumerates resonances as shown in Fig. 4). It is interesting that the dipole resonance which leaks aside from the other cylinder bears features of the both families. When the leakage from the first cylinder is directed to the second cylinder, the overlapping (15) exceeds the coupling of the Mie dipole resonant modes which leakage aside the the cylinders. As a result in the first case the resonances consequently bypass the Mie resonances while in the second case the resonances are bound to the dipole Mie resonance of isolated cylinder. For variation of the distance the $Q$ factor shows oscillating behavior with maxima which can exceed the $Q$ factor of the isolated cylinder three times. That enhancement is typical for all types of resonances except the monopole resonance which demonstrates enhancement by one order in magnitude. As one could see from Fig. 13 an observation of the resonances as dependent on the distance between the dielectric resonators needs in high quality dielectric materials with extremely low material losses.

\section{Discussions}

The recept to enhance the $Q$ factor by means of the avoided crossing of resonances is well known. Friedrich and Wintgen [3] were the first who investigated the quantitative influence of the interference of resonances on their positions and widths. Moreover, in the framework of two-level effective Hamiltonian they found out that one of the widths can turn to zero to identify the BIC. A single isolated dielectric particle of finite dimensions can not trap light because of the infinite number of radiation continua or diffraction channels [10]. However, for sufficiently large refractive index the particle shows distinctive resonances with the Q-factors which can be substantially enhanced owing to the avoided crossing of the resonances under variation of the aspect ratio of the disk $[14,15]$. Technologically, it might be challengeable to vary the size of the disk in the optical range. In the present paper we propose to vary the distance between two coaxial disks that is preferable from the experimental viewpoint. Continuous variation of the distance gives rise to an avoided crossing of the resonances due to interaction between the disks through radiating resonant modes.

Although in the present paper we considered only dielectric cylinders and disks, it is clear that the phenomenon of the avoided crossing and respective enhancement of the $Q$ factor would occur with particles of arbitrary shape when the distance between them is varied. The case of two coaxial disks simplifies computations because the solutions with different angular momentum $m$ are independent. In the present paper we have presented only the case $m=0$ because of a possibility to consider separately $\mathrm{E}$ and $\mathrm{H}$ polarizations. That case is especially preferable because of experimental setup in the form of two loop antennas coaxial to the disks. That setup was successfully used for observation of the symmetry protected BICs in long periodic array of ceramic disks [11].

\section{Acknowledgement}

We acknowledge discussions with A.A. Bogdanov and

D.N. Maksimov. This work was partially supported by RFBR grant 19-02-00055 and Ministry of Education and Science of Russian Federation (state contract $\mathrm{N}$ 3.1845.2017/4.6). 


\section{References}

[1] A.I. Kuznetsov, A.E. Miroshnichenko, M.L. Brongersma, Yu.S. Kivshar and B. Luk'yanchuk, "Optically resonant dielectric nanostructures," Science, vol. 354, pp. 2472, 2016. doi:10.1126/science.aag2472.

[2] K. Koshelev, A. Bogdanov, and Yu. Kivshar, "Metaoptics and bound states in the continuum," Science Bull., vol. 64, no. 12, pp. 836-842, 2018. doi: 10.1016/j.scib.2018.12.003.

[3] H. Friedrich and D. Wintgen, "Interfering resonances and bound states in the continuum," Phys. Rev. A, vol.32, no. 6, pp. 3231-3242, 1985. doi: 10.1103/physreva.32.3231.

[4] A.F. Sadreev, E.N. Bulgakov, and I. Rotter, "Bound states in the continuum in open quantum billiards with a variable shape," Phys. Rev. B, vol.73, no. 23, pp. 235342, 2006. doi: 10.1103/PhysRevB.73.235342.

[5] E.N. Bulgakov and A.F. Sadreev, "Bound states in the continuum in photonic waveguides inspired by defects," Phys. Rev. B, vol. 78, no. 7, pp. 075105, 2008. doi: 10.1103/PhysRevB.78.075105.

[6] S.P. Shipman and S. Venakides, "Resonant transmission near non robust periodic slab modes," Phys. Rev. E, vol. 71, no. 2, pp. 026611, 2005. doi: 10.1103/PhysRevE.71.026611.

[7] D. C. Marinica, A. G. Borisov, and S.V. Shabanov, "Bound States in the Continuum in Photonics," Phys. Rev. Lett., vol.100, no. 18, pp. 183902, 2008. doi: 10.1103/PhysRevLett.100.183902.

[8] Chia Wei Hsu, Bo Zhen, J. Lee, Song-Liang Chua, S.G. Johnson, J.D. Joannopoulos, and M. Soljačić, "Observation of trapped light within the radiation continuum," Nature, vol.499, pp. 188-191, 2013. doi: 10.1038 /nature 12289 .

[9] E.N. Bulgakov and A.F. Sadreev, "Bloch bound states in the radiation continuum in a periodic array of dielectric rods," Phys.Rev. A, vol. 90, no. 5, pp. 053801, 2014. doi: 10.1103/PhysRevA.90.053801.

[10] E.N. Bulgakov and A.F. Sadreev, "Bound states in the continuum with high orbital angular momentum in a dielectric rod with periodically modulated permittivity," Phys. Rev. A, vol. 96, no. 1, pp. 013841, 2017. doi:10.1103/PhysRevA.96.013841.

[11] Z.F. Sadrieva, M.A. Belyakov, M.A. Balezin, P.V. Kapitanova, E.A. Nenasheva, A. F. Sadreev, and A.A. Bogdanov, "Experimental observation of a symmetryprotected bound state in the continuum in a chain of dielectric disks," Phys. Rev. A, vol. 99, no. 5, pp. 053804, 2019. doi: 10.1103/physreva.99.053804.
[12] E.N. Bulgakov and A.F. Sadreev, "High- Q resonant modes in a finite array of dielectric particles, Phys. Rev. A, vol. 99, no. 3, pp. 033851, 2019. doi: 10.1103/physreva.99.033851.

[13] A. Taghizadeh and Il-Sug Chung, "Quasi bound states in the continuum with few unit cells of photonic crystal slab," Appl. Phys. Lett., vol. 111, no. 3, 031114, 2017. doi: 10.1063/1.4990753.

[14] M.V. Rybin, K.L. Koshelev, Z.F. Sadrieva, K. B. Samusev, A. A. Bogdanov, M. F. Limonov and Yu. S. Kivshar, "High-Q Supercavity Modes in Subwavelength Dielectric Resonators," Phys. Rev. Lett., vol. 119 , no. 24 , pp. 243901 , 2017. doi: 10.1103 /physrevlett.119.243901.

[15] A.A. Bogdanov, K.L. Koshelev, P.V. Kapitanova, M.V. Rybin, S.A. Gladyshev, Z.F. Sadrieva, K.B. Samusev, Yu.S. Kivshar, and M. F. Limonov, "Bound states in the continuum and Fano resonances in the strong mode coupling regime," Adv. Photonics, vol. 1, no. 1, pp. 016001, 2019. doi: 10.1117/1.ap.1.1.016001.

[16] J. Wiersig, "Formation of long-lived, scarlike modes near avoided resonance crossings in optical microcavities,” Phys. Rev. Lett., vol. 97, no. 25 , pp. 253901, 2006. doi: 10.1103/PhysRevLett.97.253901.

[17] S. V. Boriskina, "Theoretical prediction of a dramatic Q-factor enhancement and degeneracy removal of whispering gallery modes in symmetrical photonic molecules," Opt. Lett., vol. 31, no. 3, pp. 338-340, 2006. doi: 10.1364/ol.31.000338.

[18] S.V. Boriskina, "Coupling of whispering-gallery modes in size-mismatched microdisk photonic molecules," Opt. Lett., vol. 32, no.11, pp. 1557-1559, 2007. doi: 10.1364/ol.32.001557.

[19] J. Unterhinninghofen, J. Wiersig, and M. Hentschel, "Goos-Hanchen shift and localization of optical modes in deformed microcavities," Phys. Rev. E, vol. 78, no. 1, pp. 016201, 2008. doi: 10.1103/PhysRevE.78.016201.

[20] M. Benyoucef, J.-B. Shim, J. Wiersig, and O. G. Schmidt, "Quality-factor enhancement of supermodes in coupled microdisks," Opt. Lett., vol. 36, no. 8, pp. 1317-1319, 2011. doi: 10.1364/ol.36.001317.

[21] G.O. Olaofe, "Scattering by Two Cylinders," Radio Science, vol. 5, no. 11, pp. 1351-1360, 1970. doi: 10.1029/rs005i011p01351.

[22] J.W. Young and J.C. Bertrand, "Multiple scattering by two cylinders," J. Acoust. Soc. Am., vol. 58, no. 6, pp. 1190-1195, 1975. doi: 10.1121/1.380792. 
[23] Tak-Goa Tsuei and P.W. Barber, "Multiple scattering by two parallel dielectric cylinders," Appl. Opt., vol. 27, no. 16, pp. 3375-3381, 1988. doi: 10.1364/ao.27.003375.

[24] K. Yasumoto and H. Jia, Modeling of Photonic Crystals by Multilayered Periodic Arrays of Circular Cylinders, in book Electromagnetic Theory and Applications for Photonic Crystals, MIT Press, Cambridge, 2006.

[25] M. Abramowitz and I.A. Stegun, Handbook of Mathematical Functions, National Bureau of Standards, 1964.

[26] I. Awai and Y. Zhang, "Coupling coefficient of resonators - An intuitive way of its understanding," Electronics and Communications in Japan (Part II: Electronics), vol. 90, no. 9, pp. 11-18, 2007. doi: 10.1002/ecjb.20342

[27] S.Y. Elnaggar, R.J. Tervo, and S.M. Mattar, "General expressions and physical origin of the coupling coefficient of arbitrary tuned coupled electromagnetic resonators,” J. Appl. Phys., vol. 118, no. 19, pp. 194901, 2015. doi: 10.1063/1.4935634.

[28] A. Tayebi and S. Rice, "Superradiant and Dark States in Non-Hermitian Plasmonic Antennas and Waveguides," arXiv: 1812.10057v1, 2018.

[29] V. G. Bordo, "Model of Fabry-Pérot-type electromagnetic modes of a cylindrical nanowire," Phys. Rev. B, vol. 81 , no. 3 , pp. 035420 , 2010. doi: 10.1103 /physrevb.81.035420.

[30] J.D. Joannopoulos, R.D. Meade, and J.N. Winn, Photonic Crystals: Molding the Flow of Light, Princeton Univ.Press, 1995.

[31] P. Lalanne, Wei Yan, K. Vynck, C. Sauvan, and J.-P. Hugonin, "Light Interaction with Photonic and Plasmonic Resonances," Laser \& Photonics Reviews, vol.12, no. 5, pp. 1700113, 2018. doi: 10.1002/lpor.201700113.

[32] R.M. More and E. Gerjuoy, 'Properties of Resonance Wave Functions," Phys. Rev. A, vol. 7, no. 4, pp. 1288-1303, 1973. doi: 10.1103/physreva.7.1288.

[33] E.A. Muljarov, W. Langbein, and R. Zimmermann, "Brillouin-Wigner perturbation theory in open electromagnetic systems," Europhys. Lett., vol. 92, no. 5, pp. 50010, 2010. doi: 10.1209/0295-5075/92/50010.

[34] A.W. Snyder and J.D. Love, Optical Waveguide Theory, Springer, 1984.

[35] H.H. Li, "Refractive Index of Silicon and Germanium and Its Wavelength and Temperature Derivatives," J. Phys. Chem. Ref. Data, vol. 9, pp. 561-658, 1993.
[36] A. Abdrabou and Ya Yan Lu, "Exceptional points for resonant states on parallel circular dielectric cylinders," J. Opt. Soc. Am. B, vol. 36, no. 6, pp. 16591667, 2019. doi: 10.1364/josab.36.001659. 